Title: Cooperative chemical-mechanical interactions during ion exchange promote rotational ordering in hydrated montmorillonite

Authors: Michael L. Whittaker, ${ }^{1,2}$ Laura N. Lammers, ${ }^{3}$ Sergio Carrero, ${ }^{1,2}$ Benjamin Gilbert, ${ }^{1}$ Jillian F. Banfield $^{1,2 *}$

${ }^{1}$ Lawrence Berkeley National Laboratory, Earth and Environmental Sciences Division, Berkeley, California, USA.

${ }^{2}$ Department of Earth and Planetary Science, University of California, Berkeley, California, USA.

${ }^{3}$ Department of Environmental Science, Policy, and Management, University of California, Berkeley, California, USA.

*Contact: jbanfield@berkeley.edu 


\title{
Cooperative chemical-mechanical interactions during ion exchange promote rotational ordering in hydrated montmorillonite
}

Michael L. Whittaker, Laura N. Lammers, Sergio Carrero, Benjamin Gilbert, Jillian F. Banfield

\begin{abstract}
Ion exchange in clays plays a major role in water, nutrient, and contaminant storage and transport in clayrich media including soils, sediments, and suspensions. Here, we show that ion exchange between sodium and potassium in hydrated montmorillonite is a cooperative process that couples ionic transport to interlayer forces that alter mesoscale particle structures. Fluctuations in cation concentration, hydration, and basal spacing within an interlayer initiate swelling or collapse that propagates once a local concentration threshold is exceeded. Coupling between adjacent interlayers then leads to collapse or swelling throughout an entire particle. Sodium- and potassium-rich particles are found to be distinct phases with a free energy difference comparable to the thermal energy, which dynamically equilibrate through the exchange of both ions and individual montmorillonite layers. This process promotes stacking order from initially turbostratic particles via (nearly-)oriented attachment. A far more dynamic picture of clay colloids than previously expected highlights the role of nanoscale chemical-mechanical feedback in natural ion exchange phenomena.
\end{abstract}




\section{Introduction}

Clay minerals such as montmorillonite play an integral role in myriad natural processes involving $\mathrm{H}_{2} \mathrm{O}, \mathrm{CO}_{2}$, nutrient, and contaminant transport and storage in soils, sediments, and clay-rich suspensions. ${ }^{1}$ The dynamic behavior of montmorillonite arises from molecular scale interactions between 2:1 aluminosilicate layers, the counterions that balance charge arising from isomorphic substitutions within the layers, and associated waters of hydration (Figure 1). Varying the types and proportions of various interlayer species allows montmorillonite to expand and collapse reversibly, ${ }^{2}$ leading to an array of particle arrangements composed of stacks of $2: 1$ layers. ${ }^{3,4}$ The rate of solute transport, ${ }^{5}$ and the rheological and mechanical properties of soils, sediments, and suspensions ${ }^{6,7}$ are dramatically affected by clay particle structures.

Water plays a critical role in the thermodynamics and kinetics of ion-exchange in montmorillonite crystalline hydrates with either one, two, or three molecular layers of water separating the 2:1 layers. Cation binding to the montmorillonite surface is endothermic, ${ }^{8}$ and exchange is only exothermic overall when compensated by favorable cation hydration in solution, ${ }^{9}$ such that selectivity for poorly hydrated ions increases as the amount of interlayer water decreases. ${ }^{10}$ For example, exchange of potassium for more strongly hydrated sodium has a well-known tendency to promote collapse into a state of lower hydration through the expulsion of interlayer water. ${ }^{11-14}$ The presence of interlayer water also strongly affects ion-exchange rates, which are orders of magnitude slower in anhydrous phlogopites ${ }^{15}$ and illites ${ }^{16}$ than in hydrated smectites. ${ }^{17}$

A challenge for developing an improved understanding of ion exchange is that to date, neither computational or experimental approaches have been able to directly access the timescales over which both exchange and swelling/collapse occur. Molecular dynamics simulations have produced free-energy relationships for a narrow range of solution conditions ${ }^{7}$ but simulations are restricted to small system sizes and timescales. Experiments often probe equilibrium swelling states under a variety of conditions, but have not directly captured the rapid process of exchange. There is also increasing appreciation that the nanoscale details of the interactions between layers, such as crystallographically-dependent mechanical properties, ${ }^{18}$ are important to their macroscopic properties. Yet, neither simulations nor experiments have been able to explore the impact of rotation, translation or bending of layers on ion exchange.

Here, we use in situ time-resolved simultaneous small-, medium-, and wide-angle X-ray scattering (SAXS/MAXS/WAXS) to probe the dynamics of ion exchange upon rapid mixing of homoionic sodium- or potassium-montmorillonite suspensions with solutions containing the opposite cation. With a time-resolution of $\sim 100 \mathrm{~ms}$, we investigate the effects of ion exchange over three orders of spatial magnitude in reciprocal space. Transmission electron microscopy at cryogenic conditions (cryoTEM) of hydrated samples in aqua provides a complementary snapshot of particle arrangements with at least $2.2 \AA$ resolution in real space. Combined, these observations demonstrate that collapse is a phase transition that proceeds with small energy barriers accessible through thermal fluctuations. Physical separation of either collapsed or un-collapsed particles is achieved through delamination into smaller particles in the presence of potassium, enabling the development of stacking order that spans two molecular layers of water. 


\section{Results \\ Stopped-flow time-resolved X-ray scattering}

Time-resolved X-ray scattering was employed to determine the rate and mechanism by which sodium and potassium exchange following rapid mixing. Comparison to static X-ray scattering from homoionic end-members served as a basis set for interpreting data from mixed-cation solutions. Wyoming montomorillonite (SWy-2, Supporting Figure 1) was resuspended in $\mathrm{NaCl}(1 \mathrm{M})$ or $\mathrm{KCl}(1 \mathrm{M})$ to a concentration of $20 \mathrm{mg} / \mathrm{mL}$ (herein called Na-MMT or K-MMT, respectively). SAXS/MAXS/WAXS were acquired simultaneously on three separate detectors ${ }^{19}$ from both Na-MMT and K-MMT suspensions (Figure 1a, d). Comparable $(020)+(110)$ peak intensities $\left(I_{K-M M T}^{(020)+(110)} / I_{N a-M M T}^{(020)+(110)}=1.03\right.$, Materials and Methods) between Na-MMT and K-MMT confirm that the nominally identical clay concentrations yielded very similar actual concentrations of suspended layers. However, the basal spacing peak intensity at scattering vectors, $q_{001}$, of K-MMT was much lower than the intensity of Na-MMT $\left(I_{K-M M T}^{(001)} / I_{N a-M M T}^{(001)}\right.$ $=0.28$ ) and had much broader tails (Figure 1, Supporting Table 1). This indicates that there was a larger deviation from the average interlayer spacing in K-MMT and suggests that a higher fraction of the layers were poorly-stacked.

Higher order diffraction from the basal spacing peak was observed at $\ell \cdot q_{001}$, where $\ell$ is the diffraction order $(\ell=2,3,4$, and 6 for Na-MMT and $n=3,5$ for K-MMT). Integer values of $\ell$ are an indication that the interlayers are homogeneous with no interstratification. The weak $\ell=$ even peaks in $\mathrm{K}$ MMT are a result of potassium residing in close proximity to, or partially within, the silicate sheets. ${ }^{20}$ The presence of $\ell=2,3,4$ and 6 in Na-MMT suggests that sodium ions reside both near the silicate sheet and $\sim 2.5 \AA$ to either side of the interlayer midplane on average. ${ }^{20}$ Electron density, $\rho$, profiles along the stacking direction were calculated by Fourier synthesis of the diffraction peak intensities ${ }^{20}$ from MAXS data with SAXS and WAXS contributions subtracted (Figure 1b, e). These profiles are entirely consistent with intensity profiles measured directly from low-dose cryo-TEM images (discussed below) and are consistent with molecular models, which suggest that potassium makes inner-sphere complexes with ditrigonal cavities in the silicate sheet, ${ }^{10,21}$ while the more tightly bound hydration shell of sodium increases its probability of residence near the interlayer midplane. ${ }^{22-26}$

Ion exchange was performed by mixing a $\mathrm{KCl}$ solution $(1 \mathrm{M})$ with Na-MMT in four volume ratios, $r$, where $r=\left[\mathrm{K}^{+}\right] /\left[\mathrm{Na}^{+}\right]: 2,1,0.5$, and 0.33 . A constant total ion concentration was thereby maintained, minimizing the effects of changing ionic strength on species activities and MMT swelling state. Cations compensating structural charge $(\sim 5-12 \mathrm{mM})$ represent less than $2 \%$ of the total concentration of each ion (between $250-667 \mathrm{mM}$ ) in the limiting case of complete exchange. Experiments were repeated at $5^{\circ} \mathrm{C}, 15^{\circ} \mathrm{C}, 25^{\circ} \mathrm{C}$, and $35^{\circ} \mathrm{C}$ (Supporting Figures 2-3). Results were consistent across three repetitions at each condition. Solutions with higher $r$ exhibited lower overall intensity because the clay particle concentration was diluted to a greater extent, and because the X-ray absorption cross section of potassium is larger (Supporting Information).

Basal spacing peaks were broad $\left(0.05\right.$ and $0.13 \AA^{-1}$ FWHM for Na-MMT and K-MMT, respectively) compared to the separation between the peak maxima $\left(0.07 \AA^{-1}\right)$ but under all conditions the Na-MMT peak at $19.0 \AA$ decreased in intensity after mixing with $\mathrm{KCl}$ and a separate peak at $15.9 \AA$ emerged (Figure 2a-d). Allowing the peak location and shape parameters to vary during fitting confirmed that the positions of the primary basal spacing peaks did not change appreciably (Figure 2a). This is a strong indication that the collapse front within an interlayer region is sharp and/or moves rapidly on the timescale of the measurements ( $\sim 50 \mathrm{~ms}$ acquisition time). A single broadened peak with a maximum between the homoionic end members was not observed under any in situ conditions in this study, but is observed in partially dried samples (Supporting Figure 1). This excludes the possibility of extensive interstratification of collapsed and un-collapsed interlayers in situ ${ }^{27}$ which is directly supported by cryoTEM observations (below). 

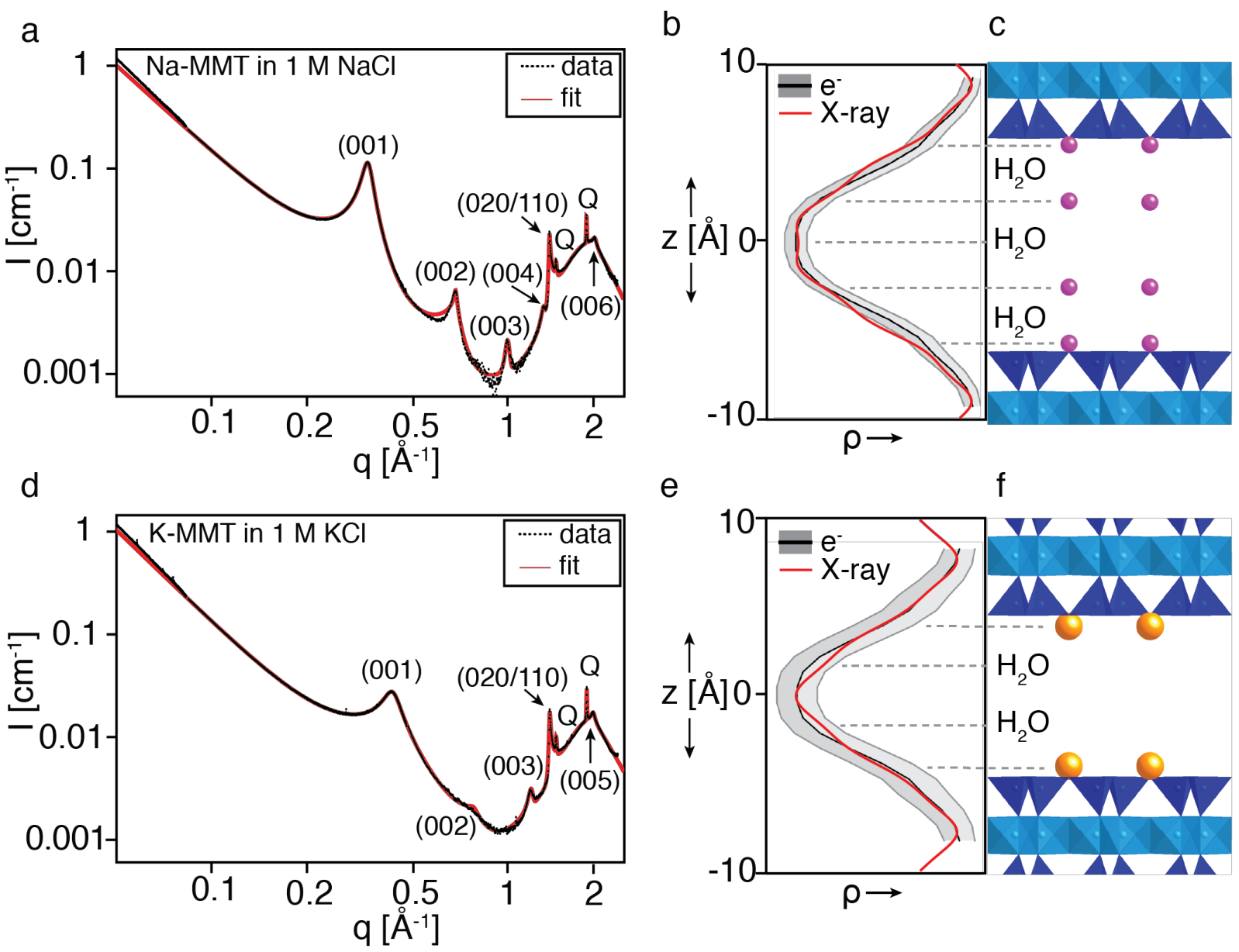

Figure 1. X-ray scattering from Na-MMT and K-MMT. (a, d) Whole-pattern SAXS/MAXS/WAXS fitting. (b, e) Electron density profiles calculated from MAXS diffraction peaks (red) or integrated TEM image contrast (black, one standard deviation in gray). (c, f) Atomic models depicting the structures inferred from the electron density profiles (interlayer water not explicitly shown).

In Na-MMT, the $\ell=2$ intensity was approximately $4 \%$ of the $\ell=1$ intensity at equilibrium (Supporting Table 1). The total noise level in the $\ell=2$ region $\left(0.56<q<0.76 \AA^{-1}\right)$ after mixing was far less (by a factor of 2.7 - 9.6) than the intensity expected in this region by taking $4 \%$ of the $19 \AA$ peak intensity (Supporting Table 2), such that this peak would be resolved if present for all but the highest mixing ratios and temperatures. Weak intensity in this region that fades after the first acquisition was often observed in such cases (Supporting Figure 4), even when collapse did not go to completion.

Weighted integrated intensity from both basal spacing peaks, expressed as the instantaneous fraction of $19 \AA$ hydrate, $f_{i}=I_{N a-M M T}^{(001)} /\left(I_{K-M M T}^{(001)}+I_{N a-M M T}^{(001)}\right)$, was used to quantify the collapse rate (Figure 2e). The total intensity from both peaks $\left(I_{K-M M T}^{(001)}+I_{N a-M M T}^{(001)}\right)$ decreases, which requires that the total coherent scattering volume decreases as collapse progresses. But the $(020)+(110)$ peak intensity remains constant, indicating that the layer concentration does not change and therefore that particles must rearrange into configurations that scatter less strongly during collapse. The $19 \AA$ A peak intensity reached an asymptotic regime of apparent equilibrium, $f$, within one second (Figure 2e) which was used to quantify the extent of collapse. 

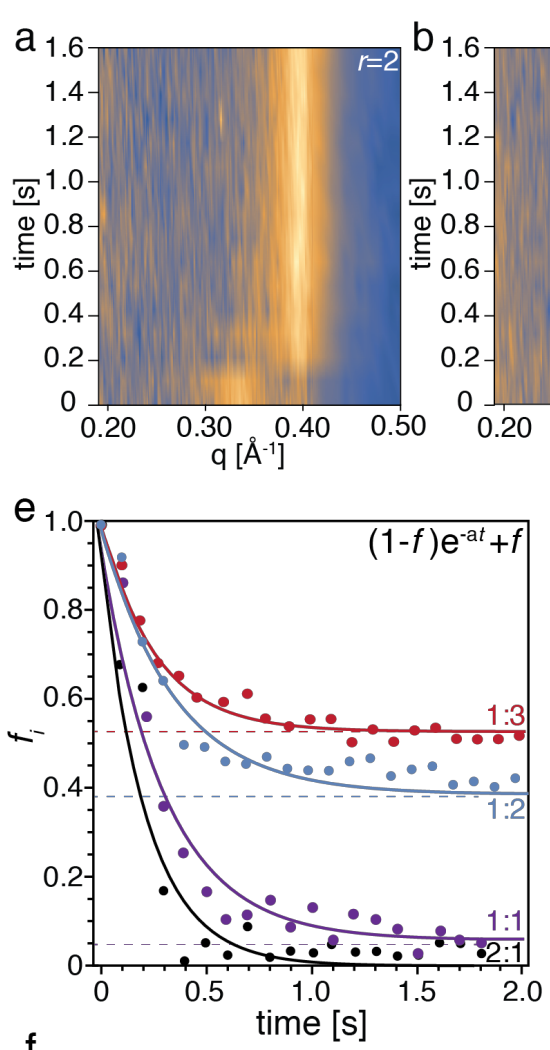

f

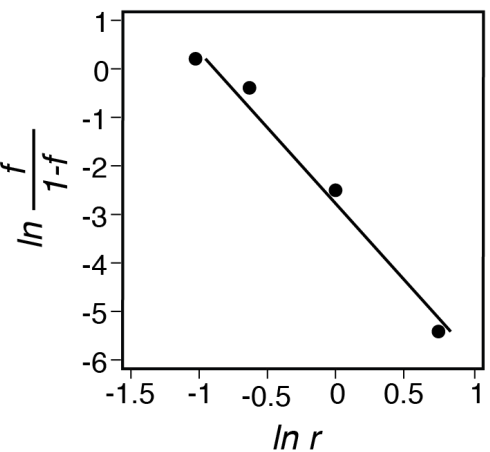

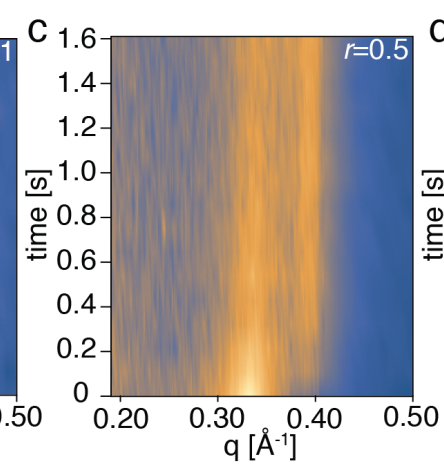
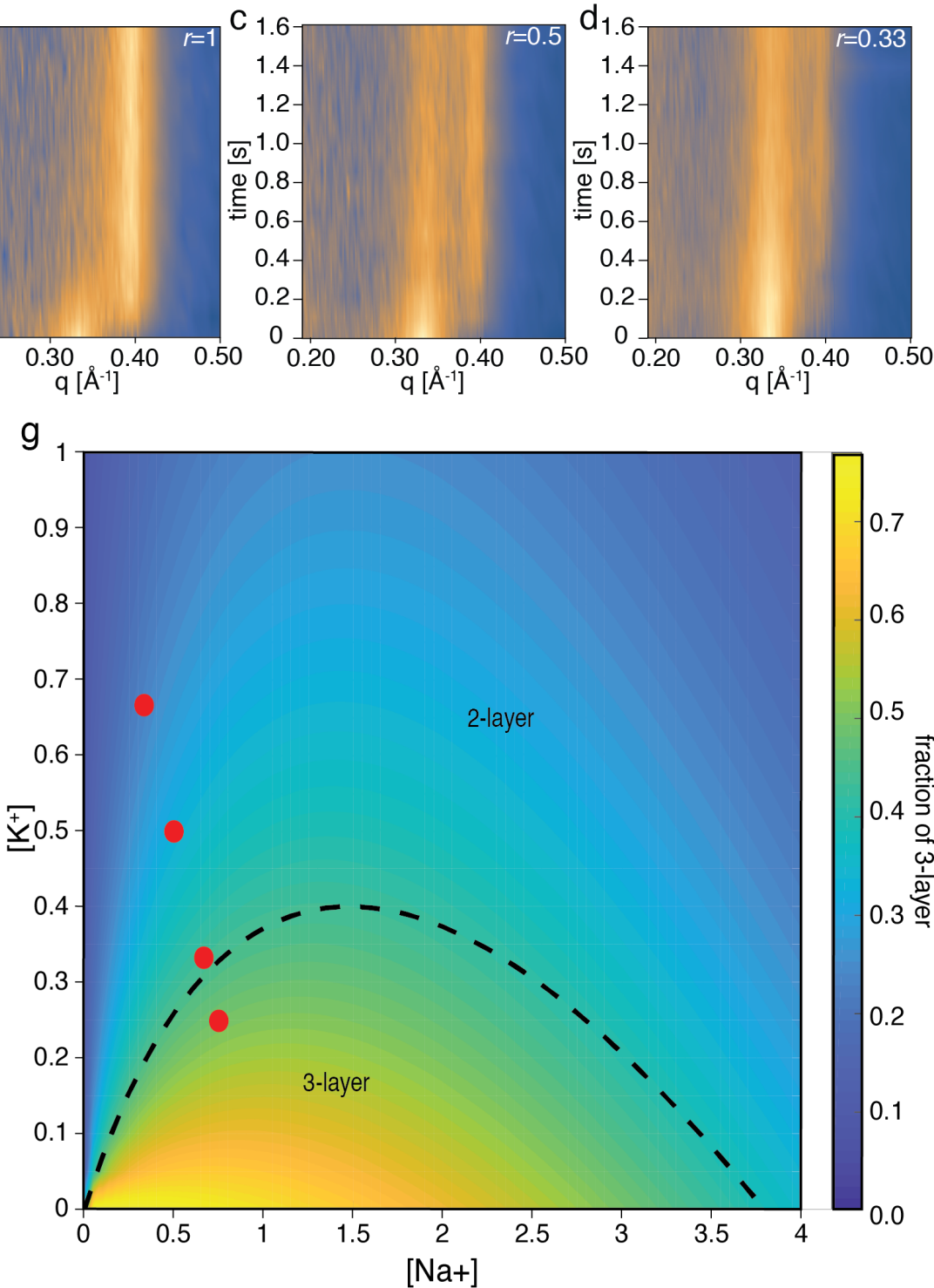

Figure 2. Collapse dynamics during ion exchange between $\mathrm{KCl}(1 \mathrm{M})$ and Na-MMT. (a-d) Emergence of a 2-layer hydrate peak at $\mathrm{q}=0.395 \AA^{-1}(15.9 \AA)$ immediately following mixing at $5^{\circ} \mathrm{C}$. (e) Time dependence of the extent of collapse, $f_{i}$, shown in (a-d). (f) Plot of $\ln \frac{f}{1-f}$ vs. $\ln r$ used to determine the equilibrium exchange constant. (g) Composition phase diagram derived using equilibrium constant calculated from (f).

A decaying exponential that accounts for the dependence of $f$ on $r$ was fit to all collapse rate data with a single parameter, $a$, that reflects both the rate of ion exchange and the rate of collapse and is thus a chemical-mechanical rate constant (Figure 2e and Equation 4, Materials and Methods). Both $f$ and $a$ were strong functions of the potassium concentration but neither exhibited a strong temperature dependence (Supporting Figure 4). This behavior contrasts with ion diffusion-limited exchange in anhydrous phyllosilicates, ${ }^{15}$ which increases appreciably with temperature through the Arrhenius behavior of the diffusion coefficient. ${ }^{28}$ Interlayer water diffusion has a strong temperature dependence, ${ }^{29}$ and partitioning between bulk solution and the interlayer is essentially barrierless, ${ }^{30}$ indicating that neither process limits collapse. The rapid collapse rate indicates that both chemical and mechanical equilibrium are reached quickly, and suggests that both two- and three-water-layer states dynamically interconvert. 


\section{Equilibrium geochemical model}

Ion exchange equilibrium between three-water-layer MMT $(\overline{\overline{\overline{M M T}}})$, two-water-layer MMT $(\overline{\overline{M M T}})$, and bulk aqueous can be expressed as

$\overline{\overline{\overline{N a_{x} K_{1-x} M M T \cdot m H_{2} \mathrm{O}}}}+n K_{a q}^{+} \Leftrightarrow \overline{\overline{N a_{x-n} K_{1-x+n} M M T}}+n N a_{a q}^{+}+m H_{2} O$

with $x$ establishing the fraction of cation sites occupied by sodium in $\overline{\overline{\overline{M M T}}}, n$ setting the composition of $\overline{\overline{M M T}}$ relative to $x$ via the stoichiometry of the exchange reaction, and $m$ water molecules per unit structural charge in the MMT layer released upon collapse. The law of mass action defines the equilibrium constant, $K_{e q}$, for this reaction as

$K_{e q}=\frac{\left(\overline{\overline{N a_{x-n} K_{1-x+n}}}\right)}{\left(\overline{\overline{N a} a_{x} K_{1-x}}\right)}\left(\frac{\left(N a^{+}\right)}{\left(K^{+}\right)}\right)^{n}\left(\frac{\overline{H_{2} O}}{\overline{\overline{H_{2} O}}}\right)^{m}$

where quantities in parentheses refer to species activities. Under the conditions employed in this study (Supporting Information), this equation simplifies to

$\ln \frac{1-f}{0.28 f}=\ln \left(K_{e q}\right)-(n+m(1-x)) \ln r$

A plot of $\ln \frac{f}{1-f}$ vs. $\ln r$ (Figure 2f) yields linear relationship that allows for the determination of $\ln \left(C K_{e q}\right)$ and $n+m(1-x)$. Commonly reported values of interlayer water density ${ }^{10,11}$ give $m \cong 10$. Thus, the composition of the solid phases, which must obey $n \leq x$ can be constrained to the range $0.76<x$ $<1$. The standard Gibbs free energy, $\Delta G^{o}$, can be determined from $K_{e q}$ such that $\Delta G^{o}<4.28 \mathrm{kT}$. This confirms that two- and three-water-layer hydrates are distinct coexisting phases in equilibrium and separated by a difference in free energy comparable to the thermal energy (Supporting Information). This model was extended to a range of sodium and potassium concentrations in the high-salinity regime, allowing for the determination of a composition-phase diagram (Figure 2g).

\section{Low-dose cryo-TEM}

Low-dose cryo-TEM imaging was employed to directly identify populations of distinct collapsed or expanded particles, reveal evidence of delamination and restacking in mixed-cation solutions, and determine the crystallographic orientation of individual layers in aqua. Rapidly quenched $r=0.5$ mixtures (Figure 4) were compared to Na-MMT and K-MMT end-members (Figure 5). Both the interlayer spacing and intralayer lattice fringes were used to characterize three-dimensional layer orientations and particle arrangements from two-dimensional images. Basal spacing measurements were obtained directly from real-space images as described for osmotic hydrates. ${ }^{31}$ Larger interlayer spacings are expected for samples frozen at cryogenic temperatures compared to aqueous samples at ambient conditions due to the expansion of vitreous ice, ${ }^{32}$ which accounts for differences between cryo-TEM and X-ray scattering data. Despite these small differences, contrast profiles measured parallel to the stacking direction were highly consistent with the electron density profiles calculated from X-ray scattering basal spacing peak intensities (Figure 1b, e).

Persistent stacking defects complicated the direct measurement of interlayer separation when layers were not perfectly coplanar (Figure 4a, b). For example, the termination of a layer within a particle led to a dislocation, around which the interlayer spacing between neighboring layers was far larger than average (Figure 4c). Integrated Fourier transform (FT) profiles of individual particles (Figure 4d) were used to account for the distribution of all such interlayer spacings (Materials and Methods). Na-MMT exhibited predominantly $19.3 \pm 2.1 \AA ̊$ interlayer spacings in integrated FTs, while K-MMT exhibited 16.4 
$\pm 2.5 \AA$ spacings (Figure 4e). Larger interlayer separation near defects produced asymmetric broadening observed on the high $q$ side of basal spacing peaks. Layers that extended past the edge of neighboring layers, which occur more frequently in particles with fewer layers, resulted in a larger fraction of the total layer volume that was not stacked in a coherently diffracting domain and therefore did not contribute to the basal spacing peak intensity.

The number of layers per particle for Na-MMT (mean \pm one standard deviation $=7.4 \pm 4.8, N=$ 982 layers from 133 particles) and K-MMT (7.2 \pm 6.1, $N=708$ layers from 98 particles) were distributed lognormally (Figure 4f). The distribution of K-MMT particle sizes exhibited a higher skewness, resulting in a modal layer number per particle (2.5) smaller than that of Na-MMT (4.4). Individual layer areas vary over four orders of magnitude (also distributed lognormally) and have irregular shapes (Supporting Figure 5). Thus, K-MMT has a smaller fraction of coherently-stacked layers. These observations are consistent with the lower basal spacing peak intensity of K-MMT for the same layer concentration observed in X-ray scattering (Figure 1) and indicate that the asymmetry of basal spacing peaks is due primarily to the disparity in layer sizes which give rise to intraparticle defects.

While $r=2$ and $r=1$ samples underwent collapse too rapidly for the cryo freezing procedure to quench the reaction, the finite $f$ at $r=0.5$, with appreciable amounts of both interlayer spacings, made this composition amenable to characterization via cryo-TEM following ion exchange. Two distinct populations of particles exhibited either $\sim 16 \AA$ or $\sim 19 \AA$ interlayer spacings (Figure 4e). Average interlayer spacings were determined from integrated FTs from 8 different particles comprising a total of 81 layers. Two particles (30 layers) retained $\sim 19 \AA$ spacing, while 6 particles (51 layers) were collapsed and had an average of $\sim 16 \AA$ interlayer spacing. The fraction of collapsed layers $(30 / 81=37 \%)$ is comparable to $f$ at this composition measured from X-ray scattering ( $48 \pm 8 \%$, Figure $2 \mathbf{b})$. Given the high fidelity for all edge-on layers in cryo-TEM, and the under-representation of K-MMT from X-ray scattering on a per-layer basis, this constitutes good agreement for $f$ between the two techniques, and confirms that two populations of non-interstratified particles with different average basal spacings coexist. 

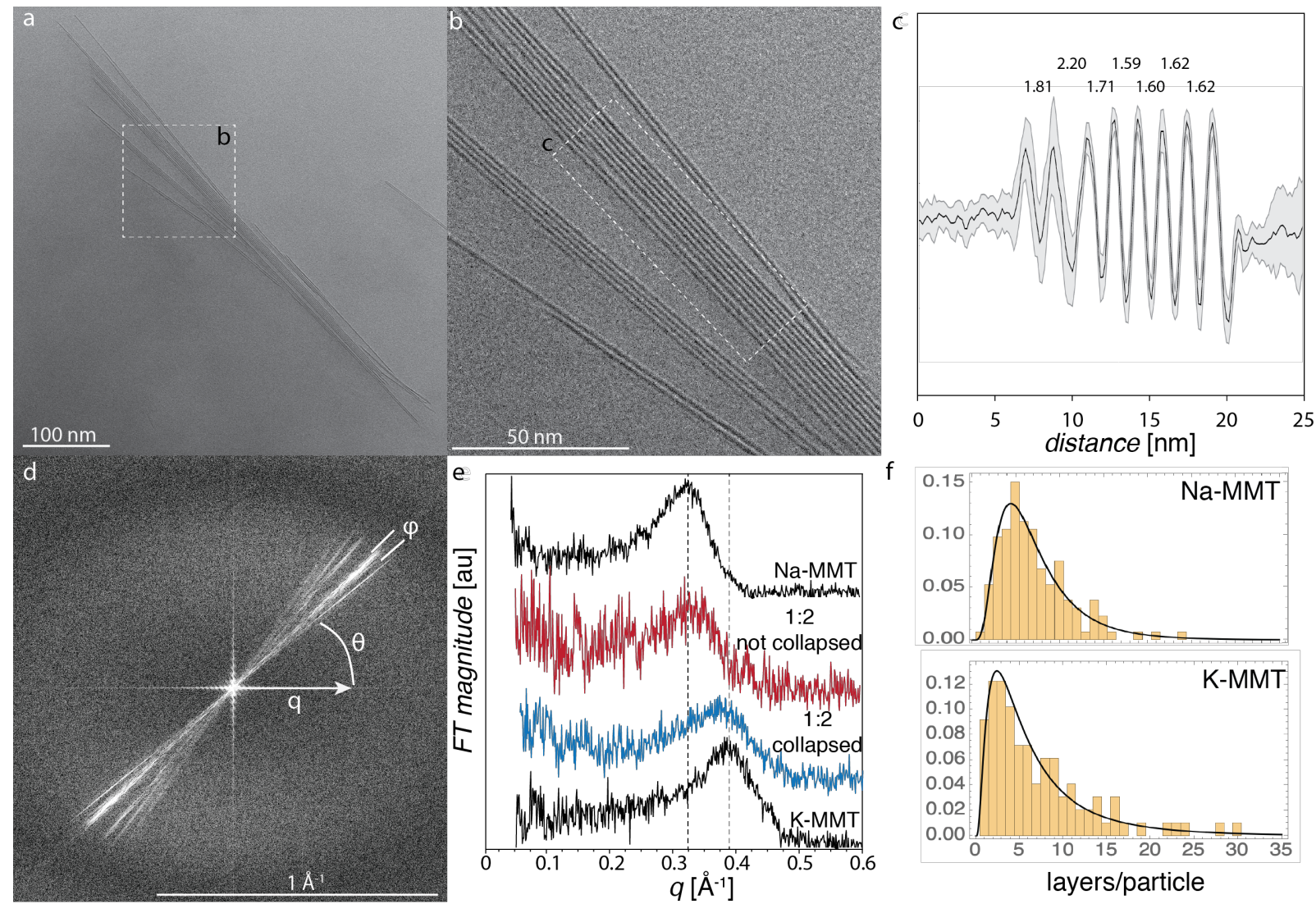

Figure 3. Cryo-TEM of 1:2 KCl:Na-MMT. (a) Low magnification image of particle. (b) Higher magnification of region indicated in (a), showing at four distinct stacking orientations from the same 'particle' comprising 16-20 layers. (c) Average intensity (one standard deviation in gray) integrated along the region indicated in (b). (d) FT of (a) (e) Comparison of the FT magnitude integrated along $q$ for a given angular range $\varphi$ for Na-MMT, K-MMT, and either collapsed or uncollapsed particles in the 1:2 sample. (f) Histograms of number of layers per particle for NaMMT and K-MMT.

Na-MMT particles were mostly coplanar, even preserving their spacing across gently deformed regions (Figure 5a, c, Supporting Figure 6), while K-MMT layers were often twisted, bent and frequently exhibited a distribution of orientations in a disordered aggregate (Figure 5a, d). Particles in $r=$ 0.5 mixtures exhibited partially delaminated domains consisting of more than one layer not in contact with adjacent layers along their entire length due to bending (Figure 4a, b, d). This is circumstantial evidence that particles undergo delamination/restacking as a consequence of the ion exchange process. Along with the observation of decreasing total basal spacing intensity in X-ray scattering during collapse in the presence of potassium, this strongly suggests that particles dynamically delaminate and restack in mixed-ion solutions.

Dynamic restacking upon collapse increased the fraction of crystallographically-oriented layers. Layers that were oriented edge-on with respect to the beam direction were rarely also aligned along a high-symmetry zone axis. Layers not aligned along a zone axis have no appreciable contrast variation within the layer and appear uniformly dark (Figure 5a, c). However, lattice fringes were frequently observed in regions directly adjacent to an edge-on particle, and FT of this region revealed inplane lattice spacings of $2.2-2.3 \AA$ and $4.5-4.6 \AA$, consistent with the $(040) /(220) /(\overline{2} 20)$ and $(020) /(110) /(\overline{1} 10)$ lattice planes, respectively, of individual clay layers (Figure 5). Bragg filtering of the FT by retaining only intensity associated with the spot(s) of interest, and taking the inverse FT, was used to correlate face-on lattice fringes with edge-on particles from a single image (Supporting Figure 7). 

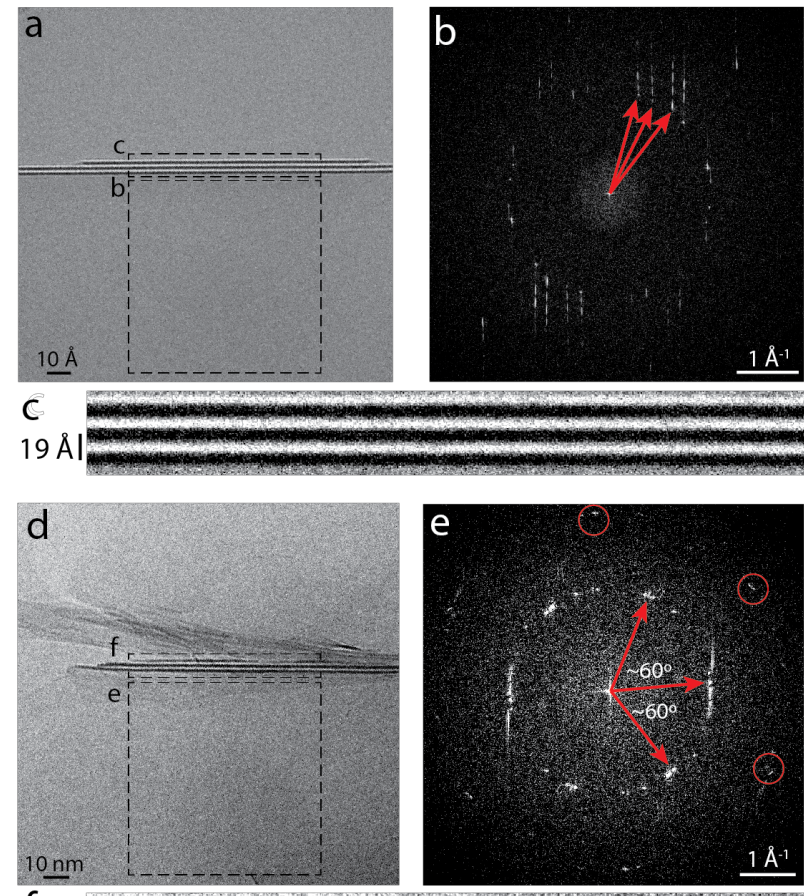

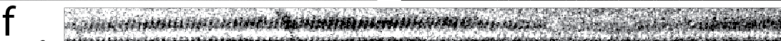

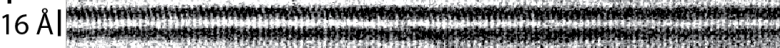

Figure 4. Cryo-TEM of Na-MMT and K-MMT. (a) Low

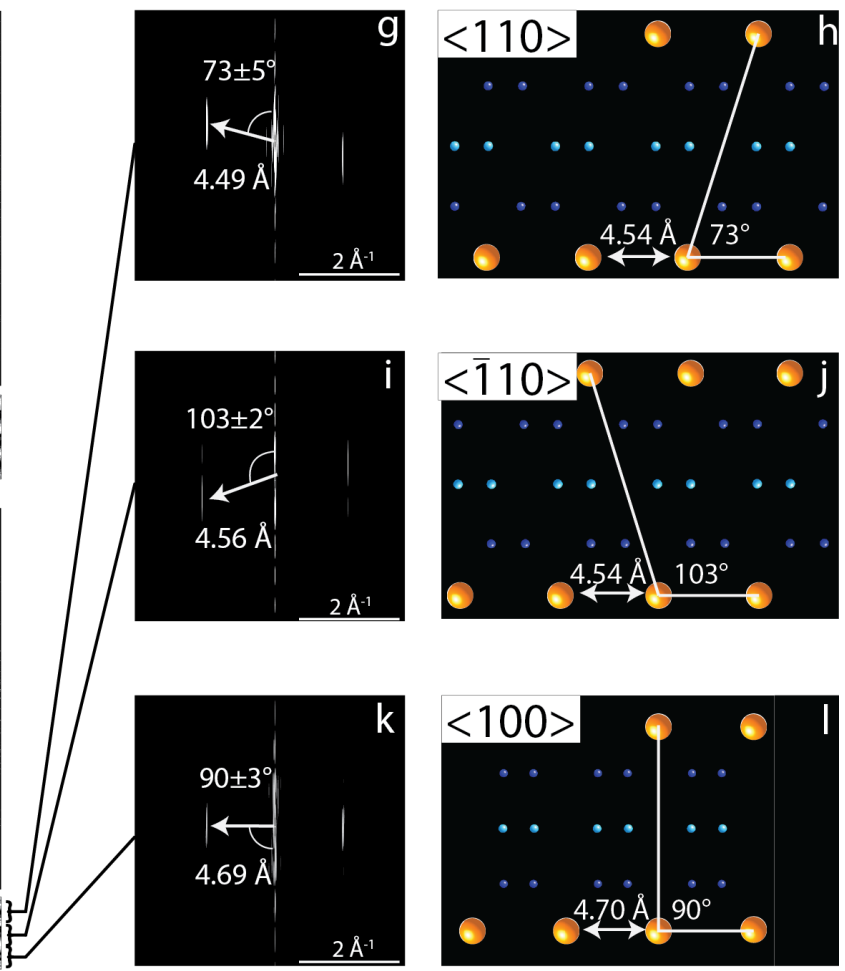
MMT particle consisting of three layers, part of which is viewed edge-on. None of the layers are oriented along a high-symmetry zone axis, but their orientation could still be determined from the FT of the adjacent region of the particle viewed face-on (b). Arrows indicate FT spots from distinct layers that are separated by $8^{\circ}$ and $13^{\circ}$ relative to each other. (c) No cross fringes are evident within the layers. (d) K-MMT particle, with three layers that are oriented edge on and many others in undetermined orientations. (e) FT spots associated with edge-on particle are clustered in $\sim 60^{\circ}$ intervals, while spots from other layers are randomly oriented. Higher order spots (circled), which are only visible for layers oriented close to face-on, confirm these relative orientations. (f) Cross fringes indicate that all three layers are in distinct orientations. (g) - (l) Fourier transform of the top, middle and bottom layer in (f), showing streaks corresponding to the projected potentials along different zone axes. The data indicate the top layer is viewed down $<110>$, the middle down $<\overline{1} 10>$ and the bottom down $<100>$.

Lattice spacings in the FT of three Na-MMT layers were found to be separated by 8 and $13^{\circ}$ (Figure 5b) and Bragg-filtering confirmed that they originate from the same layers viewed edge-on. This is consistent with turbostratic stacking commonly observed for montmorillonite. ${ }^{33}$ A fourth FT spot is identified from the Bragg filtered-FT as coming from a separate part of the image region and is not directly associated with the three-particle stack.

By chance, some Na-MMT layers were oriented along a high-symmetry axis (Supporting Figure 8). However, these layers had no special orientational relationship with their neighbors. In contrast, some K-MMT particles exhibited intra-particle lattice fringes that maintained correlated orientations across multiple neighboring layers (Figure 5d, f). For example, lattice spacings from individual layers could be resolved in three adjacent layers in the same K-MMT particle in Figure 5d-l. Fourier transformation of a narrow region containing only a single layer (Figure 5f) revealed the average spacing and angular orientation of each set of fringes (Figure 5g, i, k). Based on the angle at which the expected atomic potentials make when viewed edge-on, ${ }^{34}$ each layer was uniquely attributed to $<100>,<110>$ or $<\overline{1} 10>$ zone axes. With direct knowledge of the orientation of each layer viewed edge-on, the FT of the face-on orientation confirms that lattice spots associated with these particles were clustered at $\sim 60^{\circ}$ intervals, as expected from the lattice constants of individual layers (Figure 5e).

\section{Discussion}


These data paint a clear picture of collapse that proceeds through phase separation, resulting in particles that are non-interstratified and chemically homogeneous. This extends previous observations of phase separation between two distinct interlayer structural states ${ }^{12,35-37}$ to the case of ion exchange that is dynamic and coupled to clay swelling and collapse. We provide direct evidence that layers delaminate after potassium is introduced, as previously inferred in analogous cation exchange reactions, ${ }^{38-40}$ and suggest that this provides a mechanism for the spatial segregation of different collapse states as well as the rotation and re-attachment at (near) crystallographic orientations via Brownian motion.

Consideration of the relative stabilities of the two- and three-water-layer hydrates and the preference of these two interlayer structures for either potassium or sodium is key to understanding the process of phase separation in $\mathrm{K} / \mathrm{Na}-\mathrm{MMT}$. The observed end-member structures, with sodium in threewater-layer hydrates residing more often near the interlayer midplane and potassium in two-water-layer hydrates making inner-sphere coordination with MMT layers, correspond to local minima in free energy. ${ }^{9,10,41-43}$ Cation hydration energies are the most significant contributions to the difference. ${ }^{41,44}$ For a general solution composition, the water activity is in equilibrium with neither of these states but with a non-integer amount of interlayer water, creating a disjoining pressure. In mixed-cation systems with different hydration energies, this allows for the possibility of two coexisting basal spacings in equilibrium that are lower in energy than the mixed-cation single phase. The coexistence of potassium-rich two-waterlayer interlayers and sodium-rich three-water-layer interlayers adheres to Gibbs' phase rule (Supporting Figures 9-13). Similar behavior has been observed solutions of many mixed cations, ${ }^{45,46}$ although these data were not interpreted in the context of phase separation. The coexistence of MMT phases with different hydration states under varying relative humidity is also frequently observed in MMT systems, ${ }^{47-}$ ${ }^{50}$ including here (Supporting Figure 1).

In summary, we infer that phase-separated two- and three-water-layer interlayers are in dynamic equilibrium at an abundance (i.e., $f$ ) that is determined by the cation ratio in solution and the water activity which, in the present system, is determined by the ion concentration $(1 \mathrm{M})$ and ratio. Thus, the fraction of collapsed and un-collapsed layers, as well as their compositions, are fixed by the aqueous phase. This model is incomplete, however, without a mechanistic description of the feedback between ion exchange and collapse and the observed structural rearrangement of collapsed particles.

A striking finding of this work is that layer collapse is significantly slower than the replacement of potassium for sodium by diffusion within the interlayer. We constructed a continuum model of potassium diffusion at a 1:1 mixing ratio using the measured layer size distribution, which shows that complete intercalation is expected to occur in less than a millisecond (Supporting Figure 4). In addition, disappearance of $\ell=2$ diffraction intensity in all samples after mixing, with no appreciable change in the $\ell=1$ peak position, confirms that potassium intercalated within the interlayer before collapse initiated. Particles therefore exist in a mixed-ion configuration that is unstable with respect to phase-separated interlayers prior to collapse. That $f$ takes three orders of magnitude longer to reach an apparent equilibrium indicates that additional phenomena must be required to initiate interlayer collapse.

We propose that demixing within the interlayer is the first step in the process of collapse, and is promoted through positive feedback between the local chemical composition and the interlayer spacing. ${ }^{51}$ Regions that become enriched with potassium exhibit weaker hydration that promotes the recruitment of more potassium, and the opposite is true of sodium. ${ }^{11,12}$ Both $f$ and $a$ are largely invariant with temperature, which is consistent with the non-Arrhenian cation diffusion previously observed for MMT ${ }^{29,52}$ but inconsistent with most other processes likely to limit collapse. Thus, diffusional separation of sodium and potassium within the interlayer are required to initiate collapse.

Dynamic fluctuations within the interlayer, in which the composition, hydration state, and basal spacing are all tightly coupled, must be common and an important aspect of ion-driven swelling and collapse. Differences in hydration energy between two- and three-water-layer hydrates are 0-4 kT/unit cell of clay, with barriers of comparable size in between, ${ }^{9,10,41-43}$ suggesting that both states are regularly explored in fluctuations from the average basal spacing. ${ }^{53}$ These fluctuations may present continually changing migration barriers analogous to those responsible for non-Arrhenian behavior in ion conducting polymers. ${ }^{54}$ Therefore, fluctuations leading to a potassium-rich region may also contribute to the initiation 
of collapse. Specifically, direct measurements of interlayer spacings in cryo-TEM (Figure $\mathbf{4}$ b,c) exhibit standard deviations of approximately $15 \%$, or $\sim 2-3 \AA$, consistent with regular exploration of both hydration states with a barrier of $\sim 2-3 \mathrm{kT}$ between them.

Because potassium-saturated three-water-layer hydrates are unstable under any conditions, ${ }^{10,41} \mathrm{a}$ potassium-rich region spanning both sides of the interlayer that reaches some critical size will expel a layer of water, dramatically accelerating the recruitment of more potassium and expulsion of sodium to complete the collapse of the entire interlayer. This critical size could not be determined quantitatively here, but the concentration dependence of both $f$ and $a$ point to its existence. Extrapolating the linear increase in $f$ with decreasing potassium content below $r=1$ in Figure 3a to unity gives a potassium fraction that is larger than that at which $a$ approaches zero. In the range of concentrations between these two values, collapse is thermodynamically favored but proceeds very slowly, i.e., swelling/collapse hysteresis. This is because there is not sufficient potassium within an un-collapsed interlayer to produce potassium-rich regions that can sustain collapse via the recruitment of more potassium before fluctuations dissipate the potassium, introduce sodium, or alter the hydration state to un-collapse the region.

Based on the foregoing evidence, we propose a microscopic model of collapse outlined in Figure 6. Ion intercalation into initially homoionic Na-MMT (Figure 6a-b) is rapid but collapse is not immediate (Figure 6c-d). As the composition in the interlayer equilibrates with the surrounding solution, potassium diffuses throughout the basal surface of the MMT layer, and collapse is initiated by demixing within an interlayer. Demixing promotes potassium colocalization across both sides of an interlayer (Figure 6e-f), eventually reaching a sufficient size to overcome fluctuations and initiate collapse. Once an interlayer collapses, neighboring layers can be rarefied or collapsed, ${ }^{51}$ promoting a cascade of exchange reactions that lead to demixing of the entire particle. In some cases these layers can be liberated through delamination, and may then rotate into preferred rotational configurations via Brownian motion (Figure 6g-h).

MMT layers can stack with mica-like crystallographic order at relative orientations of only 0 or $180^{\circ}$ degrees. However, at relative particle rotations of $\sim 60^{\circ}$, neighboring particles can come into nearcrystallographic alignment along the $\langle 110\rangle,\langle\overline{1} 10\rangle,\langle 100\rangle$ directions. It has long been known that in the initial steps of illitization mica-like stacking order develops after repeated wetting and drying cycles in the presence of potassium ${ }^{55}$ resulting in this type of near-crystallographic ordering. ${ }^{56}$ This is the first demonstration that the emergence of this order first arises in suspension as a result of phase separation following potassium exchange, and is maintained across up to $6 \AA$ of water. Bending moduli of individual clay layers vary with crystallographic orientation, ${ }^{18}$ and thus, (nearly)-oriented layers are expected to be more rigid and less susceptible to perturbations through fluctuations, preserving their relative orientations. 


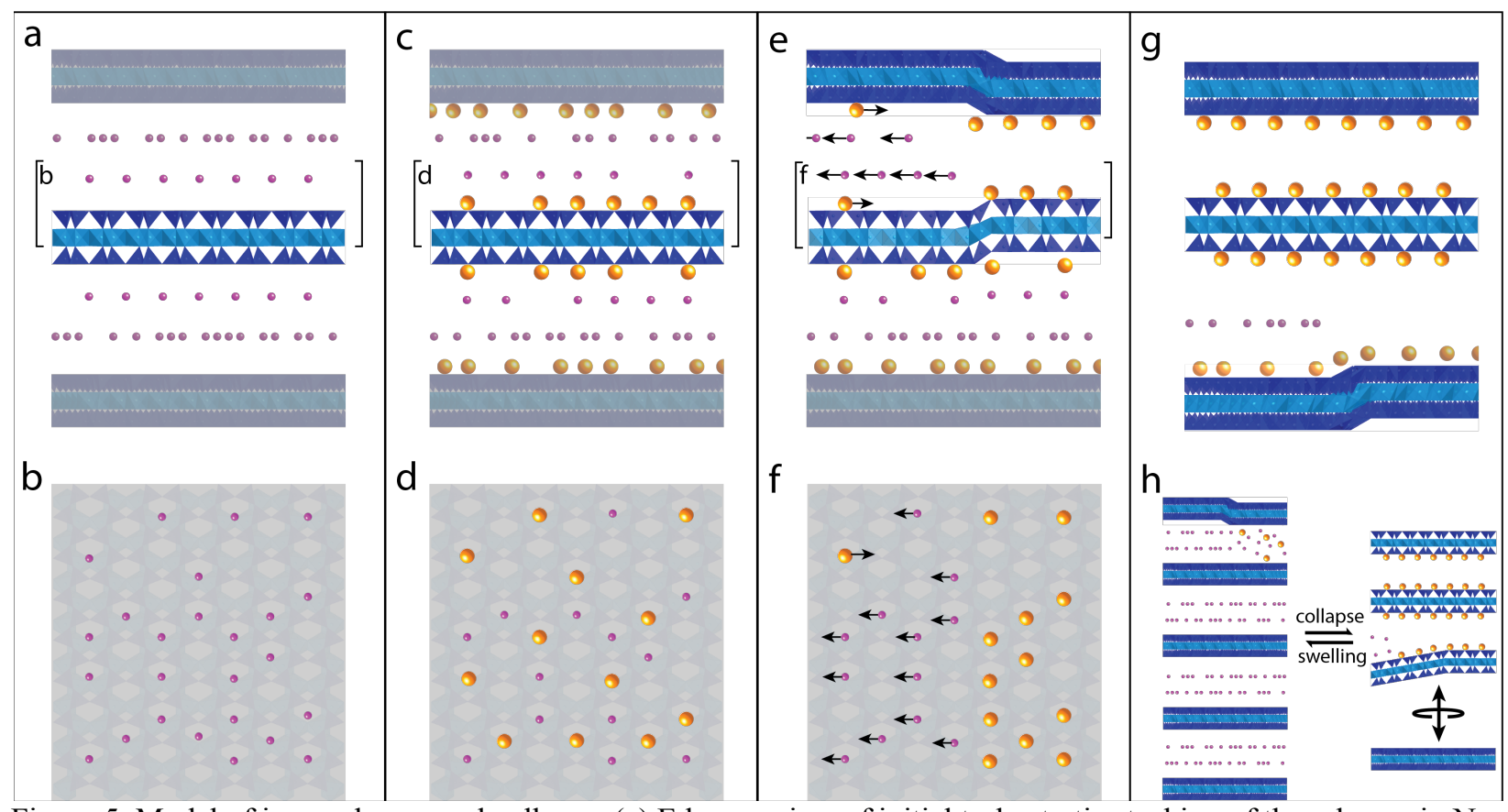

Figure 5. Model of ion exchange and collapse. (a) Edge-on view of initial turbostratic stacking of three layers in NaMMT. (b) Hypothetical distribution of sodium in proximity to the top of the middle layer, viewed face-on. (c-d) Exchange of potassium for sodium that proceeds prior to collapse. (e-f) Partial collapse induced by critical local potassium concentration. (g-h) Collapse propagates throughout layer, expelling sodium and a single layer of water. Neighboring layer becomes compressed or delaminated, allowing it to rotate into more favorable configuration.

\section{Conclusions}

Potassium exchange for sodium in montmorillonite causes initially turbostratic particles to restructure into crystallographically ordered stacking sequences. This process is initiated by the demixing of cations within the interlayer, leading to collapse of the three-water-layer hydrate via expulsion of one molecular layer of water in potassium-rich regions. Coupled interactions between layers produce two coexisting populations of collapsed or un-collapsed particles that are in chemical and mechanical equilibrium. Both collapsed and un-collaped states are accessible via thermal fluctuations, such that swelling and collapse are dynamic, and equilibrium is achieved through exchange of both cations and montmorillonite layers. This process is a clear demonstration of the nanoscale chemical-mechanical coupling that controls both the rate and products of ion exchange and the structures that result from collapse in hydrated montmorillonite.

This new understanding of exchange equilibria in which multiple swelling states are present will improve the predictive capacity of exchange models of ion binding in swelling clays. We show, for example, that a composition-phase diagram can be used to predict the coexistence of distinct swelling states, and thus the exchange selectivity, of the crystalline hydrates. We demonstrate that the reaction rate is largely unaffected by temperature, but that swelling and collapse hysteresis can be attributed to the decreasing collapse rate. An important prediction of this model is that electrolyte concentration, clay concentration, and pressure will all have a significant and quantifiable impact on the phase equilibria by altering the activity of water. While these coupled phenomena are difficult to predict using current theories of interlayer forces between clay layers, cryo-electron microscopy allows for the direct measurement of many configurational states that can be used to quantify exchange thermodynamics with lattice resolution. 


\section{Methods}

Materials

Wyoming montmorillonite (SWy-2), obtained from the Source Clays Repository of The Clay Minerals Society, was used throughout this study. An aqueous diluent phase, (herein called clay-saturated aqueous phase, CSAP) saturated with respect to SWy-2, was made by dialyzing SWy-2 (10 mg) against MilliQ water $(1 \mathrm{~L}$, resistivity $>18.2 \mathrm{M} \Omega \cdot \mathrm{cm})$ for 1 week and filtering through a $0.02 \mu \mathrm{m}$ filter (Whatman). Aqueous solutions of $\mathrm{NaCl}$ and $\mathrm{KCl}$ were prepared from reagent-grade salts and CSAP to ensure that solutions were equilibrated with respect to the clay to avoid artifacts from dissolution.

\section{Clay Treatment}

To obtain homoionic montmorillonite, SWy-2 (2-10 g) was dispersed in $\mathrm{NaCl}$ or $\mathrm{KCl}(1 \mathrm{M}, 50$ $\mathrm{mL}$ made from CSAP) in a polypropylene bottle $(50$ or $1000 \mathrm{~mL})$ and mixed on a rotating mixer $(5 \mathrm{rpm})$ for seven days. Sedimentation of coarse particles was achieved by centrifugation (1000 rcf for 10 minutes). Fine particles were separated from the coarse sediment and transferred to a cellulose dialysis membrane (Spectra/Por 3, 3.5 kDa molecular weight cutoff). Excess salt in the supernatant was removed by dialysis against CSAP, which was replaced every day for seven days. The resulting clay gel was dried in a convection oven $\left(60^{\circ} \mathrm{C}\right.$ for 14 hours, then $110^{\circ} \mathrm{C}$ for 6 hours). Suspensions were prepared by redispersing homoionic powder in either $\mathrm{NaCl}(1 \mathrm{M})$ or $\mathrm{KCl}(1 \mathrm{M})$ to a final concentration of $20 \mathrm{mg} / \mathrm{mL}$ and sonicating in an ultrasonic bath at $45^{\circ} \mathrm{C}$ for 24 hours.

\section{Time-resolved $X$-ray scattering}

X-ray scattering was performed at beamline 5ID-D of the Advanced Photon Source at Argonne National Laboratory. Small-, medium-, and wide-angle X-ray scattering (SAXS/MAXS/WAXS) was collected simultaneously on three Rayonix charge-coupled device (CCD) detectors with sample-detector distances of 8505.0, 1012.1, and $199.5 \mathrm{~mm}$, respectively. Sample-detector distances, orientations, and diffraction line broadening were calibrated using a silicon grating $(7200 \mathrm{gr} / \mathrm{mm})$, silver behenate, and lanthanum hexaboride standards, respectively, and the absolute intensity was calibrated with a glassy carbon standard. The wavelength of radiation was set to $1.2398 \AA(10 \mathrm{keV})$, resulting in a continuous range of scattering vector, $q=0.017-4.2 \AA^{-1}$. Two dimensional scattering patterns were corrected for varying incident beam intensity and sample transmission with a $\mathrm{CdWO}_{4}$ diode and azimuthally averaged using GSAS-II. ${ }^{57,58}$ Absolute scattering intensity, $I(q)$, of the clay suspensions was obtained by subtracting the average of 50 frames acquired from MilliQ water and the quartz capillary $(1.0 \mathrm{~cm}$ outer diameter $)$ from each scattering pattern.

Time-resolved acquisition was enabled by a stopped-flow mixing device (Biologic SFM400, controlled with Bio-kine software). Flow rates between 1-2 mL/s for mixing 250-500 $\mu \mathrm{L}$ of solution through Berger-Ball mixers resulted in dead times between 18.3-36.6 ms, representing a transient mixing stage. Exposure times of $0.5 \mathrm{~s}$ at $1 \mathrm{~s}$ intervals $(2 \mathrm{x} 2$ pixel binning) or $0.025 \mathrm{~s}$ at $0.050 \mathrm{~s}$ intervals $(6 \mathrm{x} 6$ pixel binning) set the minimum time resolution during the stopped-flow stage. Turbulent flow of these small volumes was confirmed to yield perfect mixing during the transient stage (before the start of data acquisition) by comparing the absolute scattering intensity from MilliQ water, $1 \mathrm{M} \mathrm{KCl}$, and mixtures of these two solutions in 2:1, 1:1, 1:2 ratios. Following each measurement, the sample was cleared from the capillary with five volumes of water $(\sim 0.5-1 \mathrm{~mL})$, and the capillary was cleaned with five volumes of water, five volumes of alcohol, and dried with air in between samples. Each experiment was repeated three times, and the results were reproducible across each run.

Rapid mixing of Na-MMT with a separate solution of $\mathrm{NaCl}(1 \mathrm{M})$ had no measurable impact on scattering intensities at any length scale investigated (Supporting Figure 2). Invariance of the scattering in the SAXS region immediately after mixing, which is sensitive to the distribution of both the aqueous and clay phases, ${ }^{4}$ suggests that mixing was complete before data acquisition commenced. Thus, any changes in scattering observed after mixing are the result of chemical processes due to ion exchange and not from turbulent flow or dilution of the MMT particles. 
The $(020)$ and (110) reflections in the WAXS region $\left(q=1-4.5 \AA^{-1}\right)$ confirmed the presence of montmorillonite as well as residual quartz that was used as an internal calibration standard. In the SAXS region $\left(q<0.1 \AA^{-1}\right)$ a Porod slope of $\sim 3$ was the dominant feature. The MAXS region $\left(1.5>q>0.1 \AA^{-1}\right)$ was characterized by the presence of a strong diffraction peak at $q_{001}=0.331 \AA^{-1}$ (19.0 $)$ for Na-MMT and $q_{001}=0.398 \AA^{-1}(15.9 \AA)$ for K-MMT, characteristic of 3- or 2-water layer crystalline hydrates, respectively. Scattering data were fit with an in-house code written in Mathematica. A Gunier-Porod fit in the SAXS region, three (K-MMT) or five (Na-MMT) (00 $\ell$ ) basal spacing peaks, Bragg peaks corresponding to the (020) and (110) MMT lattice planes and quartz (Q), and two broad Gaussains for diffuse WAXS scattering were required for a comprehensive fit to the X-ray scattering data. Fitting in the SAXS region was performed using a modified Guinier-Porod model ${ }^{59}$ for thin platelets where the scattering intensity $I$ at scattering vector $q=4 \pi \sin (\theta) / \lambda)$, takes the form

$$
I(q \ll 1)=A e^{-\frac{(q R g)^{2}}{3}} q^{-P}
$$

where $A$ is a pre-exponential intensity factor for the particle radius of gyration $R_{g}$ and $P$ is the Porod slope. The radius of gyration, $R_{g}$, defined as $R_{g}{ }^{2}=3 / 2 R^{2}$, was fixed at $560 \AA$ based on the average particle radius calculated from AFM measurements of the particle size distribution (Supporting Figure 7). A Porod slope of $P=-2.85$ for Na-MMT and -2.93 for K-MMT low $q$ (Supporting Table 1) is characteristic of lamellar structures separated by interlayer water, ${ }^{4}$ with K-MMT slightly denser, as expected for a smaller basal spacing.

Non-negligible peak asymmetry to the low- $q$ side of the basal spacing peak has been frequently observed, ${ }^{60,61}$ but has been attributed primarily to Scherrer-type broadening. Cryo-TEM images confirm that defects, primarily the termination of layers within a stack, give rise to spacings that are larger than the average interlayer separation in the vicinity of the termination (Supporting Figure 8). Radially integrated FT magnitudes from cryo-TEM images are in excellent agreement with X-ray profiles. Here, we introduce a new peak profile function that was employed to fit the basal spacing peaks and higher order reflections. This function was necessary to fit the very broad tails of the peak profiles of both potassiumand sodium-saturated montmorillonite and low- $q$ asymmetry due to defective stacking motifs measured in cryo-TEM. The diffraction intensity, $I$, in the vicinity of a diffraction peak at $q_{d}$ is given by

$$
I(q)=C\left[1+\frac{(q-\mu)^{2}}{\sigma *\left(\frac{2 \gamma}{1+e^{\alpha(q-\mu)}}\right)^{2}}\right]^{-\sigma}
$$

were $\mu$ is the peak position, $\sigma$ determines the extent of the tails, $\gamma$ is a shape parameter, $\alpha$ defines the degree of asymmetry, and $C$ is a constant proportional to the total intensity. This function yielded higherquality fits than Gaussian, Lorentzian, Pseudo-Voigt, Skewed-Gaussian, or combinations thereof.

The collapse rate of Na-MMT during ion exchange with $1 \mathrm{M} \mathrm{KCl}$ was quantified using the integrated basal spacing peak intensities $q_{001, N a-M M T}=0.332 \AA^{-1}$ and $q_{001, K-M M T}=0.400 \AA^{-1}$. The fraction of Na-MMT peak intensity contributing to the total basal spacing peak intensity, $f$, was plotted as a function of the reaction time. A renormalized exponential function was fit to extract the collapse rate, $a$, and equilibrium ratio of Na-MMT to K-MMT, $b$,

$$
f_{i}=(1-f) e^{-a t}+f
$$

\section{Cryo-transmission electron microscopy}

Suspensions of Na-MMT or K-MMT with particle concentrations of $5 \mathrm{mg} / \mathrm{mL}$ were deposited as 2-3 $\mu \mathrm{L}$ aliquots onto 300-mesh lacy carbon $\mathrm{Cu}$ grids (Electron Microscopy Sciences) which had been glow-discharged in air plasma for 15 seconds. For samples at a 1:2 mixing ratio of KCl:Na-MMT, $2 \mu \mathrm{L}$ 
Na-MMT was applied to the grid followed by $1 \mu \mathrm{L} \mathrm{KCl}$, and the resulting drop was mixed with a pipette tip for $\sim 1 \mathrm{~s}$. Excess solution was removed by automatic blotting (1 blot for $10 \mathrm{~s}$, blot force 10 at $100 \%$ relative humidity) before plunge-freezing in liquid ethane using an automated vitrification system (FEI Vitrobot). Vitrified samples were loaded into the microscope using a custom-made cryo-holder held at an operating temperature of approximately $-180^{\circ} \mathrm{C}$.

Imaging was performed with a Titan Krios TEM operated at $300 \mathrm{kV}$, equipped with a BIO Quantum energy filter. Images were recorded on a Gatan K2 IS direct electron detecting camera with a pixel size of $0.72 \AA ̊$ /pixel. Imaging was performed under cryogenic conditions using a low electron dose rate $\left(<10 \mathrm{e}^{-} \AA^{-2} \mathrm{~s}^{-1}\right)$ to minimize sample damage. Automated low-dose acquisition using SerialEM software, analogous to that used for single-particle analysis of biomacromolecules, allowed for highthroughput identification particles in edge-on orientation, which were then imaged at higher magnification. This method produces particles that are randomly oriented with respect to the beam direction such that most are not aligned edge-on, where strong absorption and diffraction from clay layers produces dark contrast that is proportional to the electron density. At least 10 images were acquired at each location, and MotionCor 2 software was used to correct for drift between frames before averaging frames together.

Measurements of interlayer spacing were performed using ImageJ 2.0.0 (NIH, http://rsb.info.nih.gov/ij/). Direct measurements of interlayer spacings were determined for coplanar layers. Fourier transform (FT) of the entire image region containing a particle was used to extract the average spacing by radially integrating the whole FT. Profiles for particles with only a single orientation at an angle $\theta$, were integrated within an angular range $\varphi$. These profiles are qualitatively comparable to Xray scattering spectra. Bragg filtering was performed by taking the FT of a region, isolating the Bragg spot(s) of interest using a binary mask to set all other pixel values to zero, and taking the inverse FT of the masked image (Supporting Figure 5). This technique reveals the source of Bragg features in the FT from the original image by reproducing the intensity they contribute, allowing for identification of particles within a stack (Supporting Figure 5c) vs. elsewhere in solution (Supporting Figure 5e). 


\section{References}

1 Bergaya, F. \& Lagaly, G. in Handbook of Clay Science Developments in Clay Science (2013).

2 Norrish, K. The Swelling of Montmorillonite. Nature 1, 120-134 (1954).

3 Paineau, E. et al. Aqueous suspensions of natural swelling clay minerals. 1. Structure and electrostatic interactions. Langmuir 27, 5562-5573, doi:10.1021/la2001255 (2011).

4 Morvan, M., Espinat, D., Lambard, J. \& Zemb, T. Ultrasmall- and small-angle X-ray scattering of smectite clay suspensions. Colloids and Surfaces A: Physicochemical and Engineering Aspects 82, 193-203 (1994).

5 Alt-Epping, P. et al. Benchmark reactive transport simulations of a column experiment in compacted bentonite with multispecies diffusion and explicit treatment of electrostatic effects. Computational Geosciences 19, 535-550, doi:10.1007/s10596-014-9451-x (2014).

6 Anderson, R. L. et al. Clay swelling - A challenge in the oilfield. Earth-Science Reviews 98, 201-216, doi:10.1016/j.earscirev.2009.11.003 (2010).

7 Bourg, I. C. \& Ajo-Franklin, J. B. Clay, Water, and Salt: Controls on the Permeability of FineGrained Sedimentary Rocks. Acc Chem Res 50, 2067-2074, doi:10.1021/acs.accounts.7b00261 (2017).

8 Rotenberg, B., Morel, J.-P., Marry, V., Turq, P. \& Morel-Desrosiers, N. On the driving force of cation exchange in clays: Insights from combined microcalorimetry experiments and molecular simulation. Geochimica et Cosmochimica Acta 73, 4034-4044, doi:10.1016/j.gca.2009.04.012 (2009).

9 Teppen, B. J. \& Miller, D. M. Hydration Energy Determines Isovalent Cation Exchange Selectivity by Clay Minerals. Soil Science Society of America Journal 70, doi:10.2136/sssaj2004.0212 (2006).

10 Liu, X. D. \& Lu, X. C. A thermodynamic understanding of clay-swelling inhibition by potassium ions. Angew Chem Int Ed Engl 45, 6300-6303, doi:10.1002/anie.200601740 (2006).

11 Gast, R. G. Standard Free Energies of Exchange for Alkali Metal Cations on Wyoming Bentonite. Soil Science Society of America Proceedings 33, 37 (1969).

12 Eberl, D. Alkali cation selectivity and fixation by clay minerals. Clays and Clay Minerals 28, 161-172 (1980).

13 Fletcher, P. \& Sposito, G. The Chemical Modelling of Clay/Electrolyte Interactions for Montmorillonite. Clay Minerals 24, 375-391 (1989).

14 S. Nir, D. Hirsch, J. Navrot \& Banin, A. Specific Adsorptionof Lithium, Sodium, Potassium, and Strontiumto Montmorillonite: Observations and Predictions1. Soil Science Society of America Journal 50, 40-45 (1968).

15 Sánchez-Pastor, N., Aldushin, K., Jordan, G. \& Schmahl, W. W. K+-Na+ exchange in phlogopite on the scale of a single layer. Geochimica et Cosmochimica Acta 74, 1954-1962, doi:10.1016/j.gca.2009.12.026 (2010).

16 Ruiz Pestana, L., Kolluri, K., Head-Gordon, T. \& Lammers, L. N. Direct Exchange Mechanism for Interlayer Ions in Non-Swelling Clays. Environ Sci Technol 51, 393-400, doi:10.1021/acs.est.6b04747 (2017).

17 Arndt, D., Mattei, M., Heist, C. \& McGuire, A. Measurement of Swelling of Individual Smectite Tactoids In Situ Using Atomic Force Microscopy. Clays and Clay Minerals 65, 92-103, doi:10.1346/ccmn.2017.064053 (2017).

18 Honorio, T., Brochard, L., Vandamme, M. \& Lebee, A. Flexibility of nanolayers and stacks: implications in the nanostructuration of clays. Soft Matter 14, 7354-7367, doi:10.1039/c8sm01359d (2018).

19 Weigand, S. J. \& Keane, D. T. DND-CAT's new triple area detector system for simultaneous data collection at multiple length scales. Nuclear Instruments and Methods in Physics Research Section A: Accelerators, Spectrometers, Detectors and Associated Equipment 649, 61-63, doi:10.1016/j.nima.2010.12.045 (2011). 
Moore, D. M. \& Robert C. Reynolds, J. Xray diffraction and the identificaiton and analysis of clay minerals. 2 edn, (Oxford University Press, 1997).

21 Vao-soongnern, V., Pipatpanukul, C. \& Horpibulsuk, S. A combined X-ray absorption spectroscopy and molecular dynamic simulation to study the local structure potassium ion in hydrated montmorillonite. Journal of Materials Science 50, 7126-7136, doi:10.1007/s10853-0159269-5 (2015).

22 Chang, F.-R. C., Skipper, N. T. \& Sposito, G. Computer Simulation of Interlayer Molecular Structure in Sodium Montmorillonite Hydrates. Langmuir (1995).

23 Chang, F.-R. C., Skipper, N. T. \& Sposito, G. Monte Carlo and Molecular Dynamics Simulations of Electrical Double-Layer Structure in Potassium-Montmorillonite Hydrates. Langmuir (1998).

24 Boek, E. S., Coveney, P. V. \& Skipper, N. T. Monte Carlo Molecular Modeling Studies of Hydrated Li-, Na-, and K-Smectites: Understanding the Role of Potassium as a Clay Swelling Inhibitor. J Am Chem Soc 117, 12608-12617 (1995).

25 Chang, F.-R. C., Skipper, N. T. \& Sposito, G. Monte Carlo and Molecular Dynamics Simulation of Electrical Double-Layer Structure in Potassium-Montmorillonite Hydrates. Langmuir 14, 1201-1207 (1998).

26 Bourg, I. C. \& Sposito, G. Molecular dynamics simulations of the electrical double layer on smectite surfaces contacting concentrated mixed electrolyte $(\mathrm{NaCl}-\mathrm{CaCl} 2)$ solutions. $J$ Colloid Interface Sci 360, 701-715, doi:10.1016/j.jcis.2011.04.063 (2011).

27 Ferrage, E. Investigation of smectite hydration properties by modeling experimental X-ray diffraction patterns: Part I. Montmorillonite hydration properties. American Mineralogist 90, 1358-1374, doi:10.2138/am.2005.1776 (2005).

28 Bourg, I. C. \& Sposito, G. Connecting the Molecular Scale to the Continuum Scale for Diffusion Processes in Smectite-Rich Porous Media. Environmental Science and Technology, 2085-2091 (2010).

29 Zheng, Y. \& Zaoui, A. Temperature effects on the diffusion of water and monovalent counterions in the hydrated montmorillonite. Physica A 392, 5994-6001, doi:10.1016/j.physa.2013.07.019 (2013).

30 Rotenberg, B. et al. Water and ions in clays: Unraveling the interlayer/micropore exchange using molecular dynamics. Geochimica et Cosmochimica Acta 71, 5089, doi:10.1016/j.gca.2007.08.018 (2007).

31 Gilbert, B., Comolli, L. R., Tinnacher, R. M., Kunz, M. \& Banfield, J. F. Formation and Restacking of Disordered Smectite Osmotic Hydrates. Clays and Clay Minerals 63, 432-442, doi:10.1346/ccmn.2015.0630602 (2015).

32 Svensson, P. D. \& Hansen, S. Combined Salt and Temperature Impact on Montmorillonite Hydration. Clays and Clay Minerals 61, 328-341, doi:10.1346/ccmn.2013.0610412 (2013).

33 Bergaya, F. \& Lagaly, G. Handbook of Clay Science. (Elsevier, 2013).

34 Tsipursky, S. I. \& Drits, V. A. The distribution of octahedral cations in the 2:1 layers of dioctaheral smectites studied by oblique texture electron diffraction. Clay Minerals 19, 177-193 (1984).

35 Laird, D. A. \& Shang, C. Relationship between cation exchagne selectivity and crystalline swelling in expanding 2:1 phyllosilicates. Clays and Clay Minerals 45, 681-689 (1997).

36 Wada, N., Hines, D. R. \& Ahrenkeil, S. P. X-ray-diffraction studies of hydration transitions in Na vermiculite. Physical Review B 41, 12895 (1990).

37 Silva, G. J. d., Fossum, J. O., DiMasi, E. \& Måløy, K. J. Hydration transitions in a nanolayered synthetic silicate: A synchrotron x-ray scattering study. Physical Review B 67, 094114, doi:10.1103/PhysRevB.67.094114 (2003).

38 Verburg, K., Baveye, P. \& McBride, M. B. Cation-Exchange Hysteresis and Dynamics of Formation and Breakdown of Montmorillonite Quasi-Crystals. Soil Science Society of America Journal 59, 1268-1273 (1995). 
39 Tournassat, C., Bizi, M., Braibant, G. \& Crouzet, C. Influence of montmorillonite tactoid size on $\mathrm{Na}-\mathrm{Ca}$ cation exchange reactions. Journal of Colloid and Interface Science 364, 443-454, doi:10.1016/j.jcis.2011.07.039 (2011).

40 Pils, J., Laird, D. \& Evangelou, V. Role of cation demixing and quasicrystal formation and breakup on the stability of smectitic colloids. Applied Clay Science 35, 201-211, doi:10.1016/j.clay.2006.09.014 (2007).

41 Tambach, T. J., Bolhuis, P. G., Hensen, E. J. M. \& Smit, B. Hysteresis in Clay Swelling Induced by Hydrogen Bonding: Accurate Prediction of Swelling States. Langmuir 22, 1223-1234 (2006).

42 Whitley, H. D. \& Smith, D. E. Free energy, energy, and entropy of swelling in Cs-, Na-, and Srmontmorillonite clays. $J$ Chem Phys 120, 5387-5395, doi:10.1063/1.1648013 (2004).

43 Smith, D. E., Wang, Y., Chaturvedi, A. \& Whitley, H. D. Molecular Simulations of the Pressure, Temperature, and Chemical Potential Dependencies of Clay Swelling. Journal of Physical Chemistry B 110, 20046-20054 (2006).

44 Heinz, H., Vaia, R. A. \& Farmer, B. L. Interaction energy and surface reconstruction between sheets of layered silicates. The Journal of Chemical Physics 124, 1, doi:10.1063/1.2202330 (2006).

45 Oueslati, W., Rhaiem, H. B., Lanson, B. \& Amara, A. B. H. Selectivity of Na-montmorillonite in relation with the concentration of bivalent cation $(\mathrm{Cu} 2+, \mathrm{Ca} 2+, \mathrm{Ni} 2+)$ by quantitative analysis of XRD patterns. Applied Clay Science 42, 224-227, doi:10.1016/j.clay.2008.09.010 (2009).

46 Vieillard, P., Blanc, P., Fialips, C. I., Gailhanou, H. \& Gaboreau, S. Hydration thermodynamics of the SWy-1 montmorillonite saturated with alkali and alkaline-earth cations: A predictive model. Geochimica et Cosmochimica Acta 75, 5664-5685, doi:10.1016/j.gca.2011.07.014 (2011).

47 Michot, L. J. et al. Phase Diagrams of Wyoming Na-Montmorillonite Clay. Influence of Particle Anisotropy. Langmuir 20, 10829-10837 (2004).

48 Ferrage, E., Lanson, B., Michot, L. J. \& Robert, J.-L. Hydration Properties and Interlayer Organization of Water and Ions in Synthetic Na-Smectite with Tetrahedral Layer Charge. Part 1. Results from X-ray Diffraction Profile Modeling. Journal of Physical Chemistry: C 114, 45154526 (2010).

49 Dazas, B. et al. Influence of Tetrahedral Layer Charge on the Organization of Interlayer Water and Ions in Synthetic Na-Saturated Smectites. The Journal of Physical Chemistry C 119, 41584172, doi:10.1021/jp5123322 (2015).

50 Holmboe, M., Wold, S. \& Jonsson, M. Porosity investigation of compacted bentonite using XRD profile modeling. J Contam Hydrol 128, 19-32, doi:10.1016/j.jconhyd.2011.10.005 (2012).

51 Schaettle, K., Ruiz Pestana, L., Head-Gordon, T. \& Lammers, L. N. A structural coarse-grained model for clays using simple iterative Boltzmann inversion. J Chem Phys 148, 222809, doi:10.1063/1.5011817 (2018).

52 S. Balme, M. Kharroubi, A. Haouzi \& Henn, F. Non-Arrhenian Ionic dc Conductivity of Homoionic Alkali Exchanged Montmorillonites with Low Water Loadings. Journal of Physical Chemistry C 114, 9431-9438 (2010).

53 < Christy - 1993 - Multistage diffusionless pathways for reconstructive phase transitions application to binary compounds and calcium carb.pdf $>$.

54 Druger, S. Ionic transport in polymer electrolytes based on renewing environments. Journal of Chemical Physics 100, 3979-3984, doi:10.1063/1.466331 (1993).

55 Plançon, A., Besson, G., Tchoubar, C., Gaultier, J. P. \& Mamy, J. Qualitative and Quantitative Study of a Structural Reorganization in Montmorillonite After Potassium Fixation. Developments in Sedimentology 27, 45-54 (1979).

56 Srodon, J. \& Eberl, D. Illite. Reviews in Mineralogy and Geochemistry 13, 495 (1984).

57 Toby, B. H. \& Von Dreele, R. B. GSAS-II: the genesis of a modern open-source all purpose crystallography software package. Journal of Applied Crystallography 46, 544-549, doi:10.1107/S0021889813003531 (2013). 
58 Von Dreele, R. B. Small-angle scattering data analysis inGSAS-II. Journal of Applied Crystallography 47, 1784-1789, doi:10.1107/s1600576714018366 (2014).

59 Beaucage, G. Approximations Leading to a Unified Exponential/Power-Law Approach to SmallAngle scattering. Journal of Applied Crystallography 28, 717-728 (1995).

60 Segad, M. et al. Microstructural and Swelling Properties of Ca and Na Montmorillonite: (In Situ) Observations with Cryo-TEM and SAXS. The Journal of Physical Chemistry C 116, 7596-7601, doi:10.1021/jp300531y (2012).

61 Tester, C. C., Aloni, S., Gilbert, B. \& Banfield, J. F. Short- and Long-Range Attractive Forces That Influence the Structure of Montmorillonite Osmotic Hydrates. Langmuir 32, 12039-12046, doi:10.1021/acs.langmuir.6b03265 (2016).

\section{Acknowledgements}

This research was supported by the U.S. Department of Energy, Office of Science, Office of Basic Energy Sciences, Chemical Sciences, Geosciences, and Biosciences Division, through its Geoscience program at LBNL under Contract DE-AC02-05CH11231. Portions of this work were performed at the DuPont-Northwestern-Dow Collaborative Access Team (DND-CAT) located at Sector 5 of the Advanced Photon Source (APS). DND-CAT is supported by Northwestern University, E.I. DuPont de Nemours \& Co., and The Dow Chemical Company. This research used resources of the Advanced Photon Source, a U.S. Department of Energy (DOE) Office of Science User Facility operated for the DOE Office of Science by Argonne National Laboratory under Contract No. DE-AC02-06CH11357. Data was collected using an instrument funded by the National Science Foundation under Award Number 0960140. Access to the FEI Titan Krios was provided through the BACEM UCB facility, and we thank Dan Toso and Elizabeth A. Montabana for technical assistance.

\section{Author Contributions}

M.L.W. conceived the idea, collected and analyzed data, and wrote the manuscript. S. C. assisted with Xray scattering data collection. B. G. and J. F. B. conceived the idea and wrote the manuscript.

Supplementary Information accompanies this paper

Competing financial interests: The authors declare no competing financial interests. 